\title{
Scaling of the critical slip distance in granular layers
}

\author{
Takahiro Hatano ${ }^{1}$
}

We investigate the nature of friction in granular layers by means of numerical simulation focusing on the critical slip distance, over which the system relaxes to a new stationary state. Analyzing a transient process in which the sliding velocity is instantaneously changed, we find that the critical slip distance is proportional to the sliding velocity. We thus define the relaxation time, which is independent of the sliding velocity. It is found that the relaxation time is proportional to the layer thickness and inversely proportional to the square root of the pressure. An evolution law for the relaxation process is proposed, which does not contain any length constants describing the surface geometry but the relaxation time of the bulk granular matter. As a result, the critical slip distance is scaled with a typical length scale of a system. It is proportional to the layer thickness in an instantaneous velocity change experiment, whereas it is scaled with the total slip distance in a spring-block system on granular layers.

\section{Introduction}

A natural fault has the cataclasite core zone, along which shear deformation concentrates (e.g. Engelder [1974]; Scholz [1987]). Rheology of these granular matters thus provides us an important insight in considering the nature of friction on faults from a microscopic point of view. Unfortunately, to this date, our understanding of the rheological properties of granular matter is still poor except for dilute flow to which the kinetic theory of gases can apply (e.g. Garzó and Dufty [1999] and references therein.) Thus, a computational approach has played a considerable role in investigating dense granular rheology to propose some constitutive laws for stationary shear flow (e.g. Aharonov and Sparks [2002]; Hazzard and Mair [2003]; GDR MiDi [2004]; da Cruz et al. [2005]; Jop et al. [2004]; Hatano [2007]). However, a nonstationary state is still a frontier in the sense that we do not have any constitutive laws for transient processes.

The description of transient states is particularly important in the context of seismology because an earthquake is essentially a nonstationary process. An important quantity is the critical slip distance, over which a fault looses its frictional strength with the coseismic slip [Ida, 1973], because it determines the maximum acceleration of the seismic ground motion $[A k i, 1987]$ as well as the rupture nucleation process (e.g. Ohnaka [2000]). However, regardless of its importance, we still can not explain the critical slip distance ranging from $10^{-1}$ to $1 \mathrm{~m}$, which is obtained by the seismic inversion [Ide and Takeo, 1997]. It is rather paradoxical that the critical slip distance obtained in a typical experiment is of the order

\footnotetext{
${ }^{1}$ Earthquake Research Institute, University of Tokyo, Tokyo, Japan
}

Copyright 2021 by the American Geophysical Union. $0094-8276 / 21 / \$ 5.00$ of $10^{-5} \mathrm{~m}$ [Dieterich, 1979; Scholz, 2002]. Understanding the physics that determines the critical slip distance to explain the wide gap between a natural fault and a laboratory is thus a central problem in seismology [Marone and Kilgore, 1998; Ohnaka and Shen, 1999; Ohnaka, 2003].

In this letter, by means of numerical simulation on sheared granular layers, we obtain a constitutive law that describes a nonstationary process as well as a stationary state. Using this constitutive law together with dimensional analysis, we propose a new interpretation on the scale dependence of the critical slip distance.

\section{Model}

In the following we describe the computational model of granular layers. Each grain is assumed to be sphere. The interaction between grains is described by the discrete element method (DEM) [Cundall and Strack , 1979], which is the standard model used in powder engineering and soil mechanics. Consider a grain $i$ of radius $R_{i}$ located at $\mathbf{r}_{\mathbf{i}}$ with the translational velocity $\mathbf{v}_{\mathbf{i}}$ and the angular velocity $\boldsymbol{\Omega}_{\mathbf{i}}$. This grain interacts with another grain $j$ when they are in contact; i.e. $\left|\mathbf{r}_{i j}\right|<R_{i}+R_{j}$, where $\mathbf{r}_{i j}=\mathbf{r}_{i}-\mathbf{r}_{j}$. The interaction consists of two kinds of forces, which are normal and transverse to $\mathbf{r}_{i j}$, respectively. Introducing the unit normal vector $\mathbf{n}_{i j}=\mathbf{r}_{i j} /\left|\mathbf{r}_{i j}\right|$, the normal force acting on $i$, which is denoted by $\mathbf{F}_{i j}^{(n)}$, is given by $\left[k h_{i j}+\zeta \mathbf{n}_{i j} \cdot \dot{\mathbf{r}}_{i j}\right] \mathbf{n}_{i j}$, where $h_{i j}=R_{i}+R_{j}-\left|\mathbf{r}_{i j}\right|$. In order to define the transverse force, we utilize the relative tangential velocity $\mathbf{v}_{i j}^{(t)}$ defined by $\dot{\mathbf{r}}_{i j}-\mathbf{n}_{i j} \cdot \dot{\mathbf{r}}_{i j}+\left(R_{i} \boldsymbol{\Omega}_{i}+R_{j} \boldsymbol{\Omega}_{j}\right) /\left(R_{i}+R_{j}\right) \times \mathbf{r}_{i j}$ and introduce the relative tangential displacement vector $\boldsymbol{\Delta}_{i j}^{(t)}=\int_{\text {roll }} d t \mathbf{v}_{i j}^{(t)}$. The subscript in the integral indicates that the integral is performed only when the contact is rolling; i.e., $k_{t}\left|\Delta_{i j}^{t}\right|<\mu_{e}\left|\mathbf{F}_{i j}^{(n)}\right|$ or $\Delta_{i j}^{t} \cdot v_{i j}^{t}<0$. Otherwise, the contact is said to be sliding. The expression of the tangential force depends on the state of the contact: $\mu_{e}\left|\mathbf{F}_{i j}^{(n)}\right| \mathbf{v}_{i j}^{(t)} /\left|\mathbf{v}_{i j}^{(t)}\right|$ for sliding contact and $k_{t} \boldsymbol{\Delta}_{i j}^{(t)}$ for rolling contact.

We consider a bidisperse system in order to avoid crystallization, where the diameters of the constituent particles are $0.7 d$ and $1.0 d$, respectively. The number of each grain is the same. For simplicity, we assume that the mass of these grains is the same, which is denoted by $m$. The dimensions of the system are $L \times L \times H$, where we use periodic boundary conditions along the $x$ and the $y$ axes. In the $z$ direction, there exist two rigid walls that consist of the larger particles. One of the walls is displaced along the $x$ axis at constant velocity $V$ to realize plain shear flow, where the velocity gradient is formed in the $z$ direction. These grains interact with the bulk grains via the force described above. This wall is also allowed to move along the $z$ axis so that the pressure is kept constant at $P$, while it is immobile along the $y$ axis. Namely, the $z$ dimension of the system, denoted by $H$, is a dynamic variable. The equation of motion of the wall along the $z$ axis is given as $M \ddot{H}=F_{z}-P L^{2}$, where $M$ denotes the mass of the wall and $F_{z}$ is the repulsive force given by the grains. We check that the mass of the wall does not influence the result. Hereafter we set $M=100 \mathrm{~m}$. We also confirm that the $x$ and $y$ dimensions do not influence the 
result by comparing two systems: $L=10 d$ and $20 d$. We thus adopt $L=10 d$ to save the computational time. The traction acting on the moving wall is monitored, which is denoted by $F_{x}$, so that the friction coefficient of the system is defined as $\mu \equiv F_{x} / L^{2} / P$. We do not consider gravity here.

For de-dimensionalization, we set $d=1, k=1$, and $m=1$. The other parameters are chosen as $k_{t}=k / 5$, $\mu_{e}=0.6$, and $\zeta=1$. The coefficient of restitution vanishes with these parameters, which may be justified in modeling rock powder. However, the general correspondence of these parameter values in DEM to the material constants in real life is not rigorous. Here we estimate the material constants based on the sound velocity and the Young's modulus, which are roughly estimated as $d \sqrt{k / m}$ and $k / d$, respectively. (Note that the numerical factors are neglected.) Thus, the unit velocity and the unit pressure in DEM are of the order of kilometer per second and several tens of Gigapascal, respectively.

\section{Result}

We first prepare a stationary state with the wall velocity $V_{1}$. The stationarity is checked by monitoring the friction coefficient, the volume, and the internal velocity gradient. Then the wall velocity is instantaneously switched from $V_{1}$ to $V_{2}$. The typical response of the system is shown in Figure 1 , where the friction coefficient relaxes to a new stationary value corresponding to the new sliding velocity $V_{2}$. The sharp increase of the friction coefficient at the instance of the velocity change is due to the steeper velocity gradient near the wall. Then this nonlinear velocity gradient relaxes to the uniform velocity gradient, which leads to the relaxation of the friction coefficient. The relaxation behaviors of the friction coefficient $\mu$ and the layer thickness $H$ can be fitted by the exponential curve, as is shown in Figures 1a and $\mathrm{b}$.

$$
\begin{aligned}
\mu(x) & =\mu_{2}+\left(\mu_{1}-\mu_{2}\right) \exp \left(-x / D_{c}\right), \\
H(x) & =H_{2}+\left(H_{1}-H_{2}\right) \exp \left(-x / D_{c}\right),
\end{aligned}
$$

where $x$ denotes the slip distance after the velocity switch and $D_{c}$ defines the critical slip distance. We confirm that the critical slip distance is almost the same for the friction coefficient and the layer thickness. We test several values of $V_{1}, V_{2}, P$, and $H$ to find that the choice of $V_{1}$ does not apparently affect the critical slip distance. We thus fix $V_{1}=1 \times 10^{-5}$ and change $V_{2}, P$, and $H$ in the following.

As is shown in Figure 2, the critical slip distance depends on the slip velocity $V_{2}$ and the normal pressure $P$. Importantly, the critical slip distance is proportional to the slip velocity so that the relaxation time can be defined as the proportional coefficient.

$$
D_{c} \simeq \tau V_{2}
$$

where the relaxation time $\tau$ is independent of the velocity (but still depends on the pressure). In this sense, the relaxation time is more fundamental than the critical slip distance in sheared granular layers. This makes a quite contrast to conventional experiments on friction of two surfaces, where the critical slip distance is independent of the sliding velocity (e.g. see Marone [1998]). The discrepancy is due to the different physical mechanisms of friction. In the rubbing of two surfaces, the area of true contact (asperity) determines the friction coefficient so that the critical slip distance is of the order of the typical dimension of asperities (e.g. tens of micrometers), whereas the internal velocity profile determines the friction coefficient in granular layers.

Then we discuss the nature of the relaxation time. In Figure $3 \mathrm{a}$, we find that the relaxation time is inversely proportional to the square root of the pressure.

$$
\tau \propto P^{-1 / 2} .
$$

Although it has been recognized that the intrinsic time constant in granular matter should be scaled as equation (4) from the viewpoint of dimensional analysis, this relation has not been confirmed in a dense system. It is also found that the relaxation time is proportional to the layer thickness, as is confirmed in Figure 3 a.

$$
\tau \propto H
$$

which implies that the perturbation propagates into the granular layers at the constant velocity. This makes a quite contrast to Newtonian fluids, where the velocity field is diffusive so that the relaxation time is proportional to the square of the layer thickness. However, at this point, we cannot de-

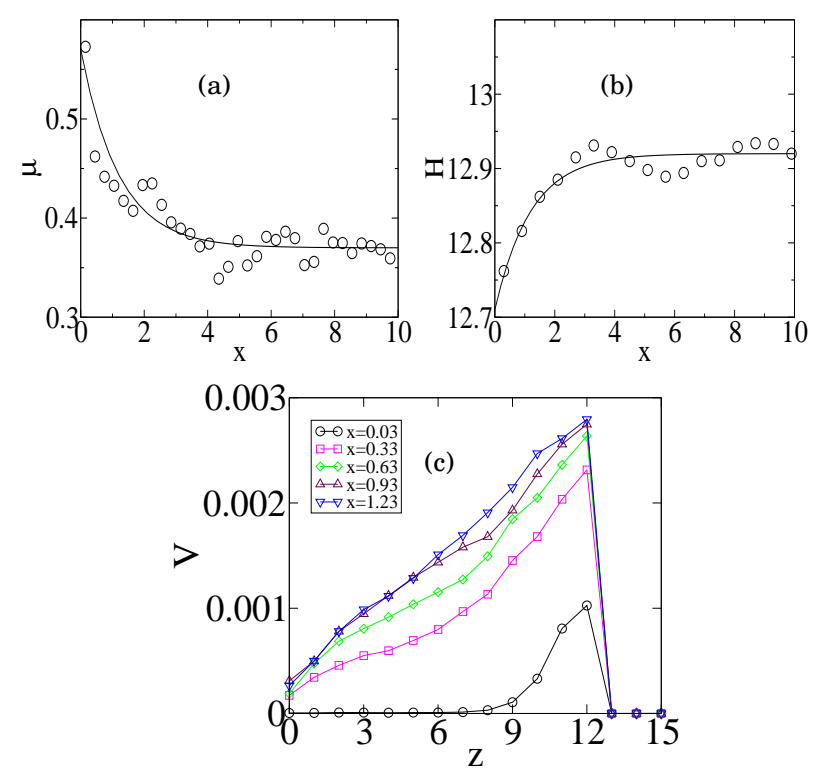

Figure 1. The relaxation behaviors of the system after the velocity change. Here $V_{1}=1 \times 10^{-5}, V_{2}=3 \times 10^{-3}$, and $P=1 \times 10^{-3}$. (a) The friction coefficient $\mu$ and (b) the layer thickness $H$. The horizontal axes represent the slip distance after the velocity change. Symbols denote the simulation data, while the solid lines denote the exponential curves, Eqs. (1) and (2). (c) Relaxation of the internal velocity profile, which is defined as the instantaneous local mean velocity in the $x$ direction.
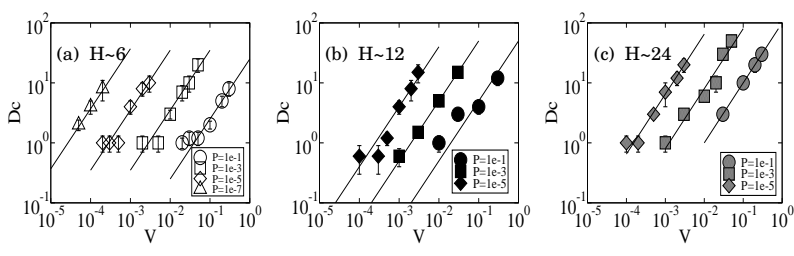

Figure 2. The critical slip distance $D_{c}$ as a function of the velocity, $V=V_{2}$. (a) $H \simeq 6 d$, (b) $H \simeq 12 d$, and (c) $H \simeq 24 d$. 
rive Eqs. (4) and (5) from the microscopic principle, i.e., the particle dynamics,

From Eqs. (4) and (5), the relaxation time reads

$$
\tau=c \frac{H}{d} \sqrt{\frac{m}{P d}} .
$$

where $c$ is a numerical factor. We find $c=1.0 \pm 0.1$ in Figure 3a. Equivalently, from equation (3), the critical slip distance can be written as

$$
D_{c}=c H V_{2} \sqrt{\frac{m}{P d^{3}}}=c I \frac{H^{2}}{d},
$$

where $I \equiv V_{2} / H \sqrt{m / P d}$ is a nondimensional number known as the inertial number (see GDR MiDi [2004] for its usefulness in describing granular rheology, while it is originally defined by Savage and Hutter [1989].) We can confirm the validity of equation (7) in Figure $3 \mathrm{~b}$. Note that the critical slip distance is proportional to the layer thickness. This is consistent with an experiment in which the critical slip distance is scaled with the gouge layer thickness [Marone and Kilgore, 1998].

Then we introduce the evolution law for the transient states. The exponential relaxation of the friction coefficient, equation (1), implies that the evolution law is a first order
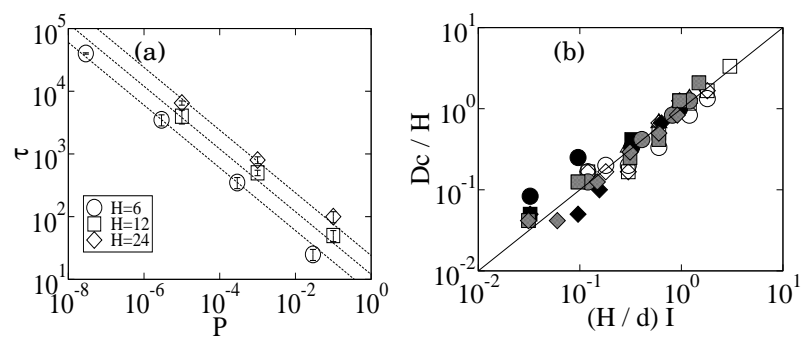

Figure 3. (a) The pressure dependence of the relaxation time, defined by equation (3). Each line denotes $\tau / P^{1 / 2}=$ $6,14,24$. (b) The critical slip distance $D c$ divided by $H$ as a function of the inertial number $I \equiv V_{2} / H \sqrt{m / P d}$ multiplied by $H / d$, i.e., $V_{2} / \sqrt{m / P d^{3}}$. Thus, this indicates that the critical slip distance is proportional to the layer thickness. The solid line represents $D_{c} / H=V_{2} \sqrt{m / P d^{3}}$. The legends are the same as those in Figure 2.
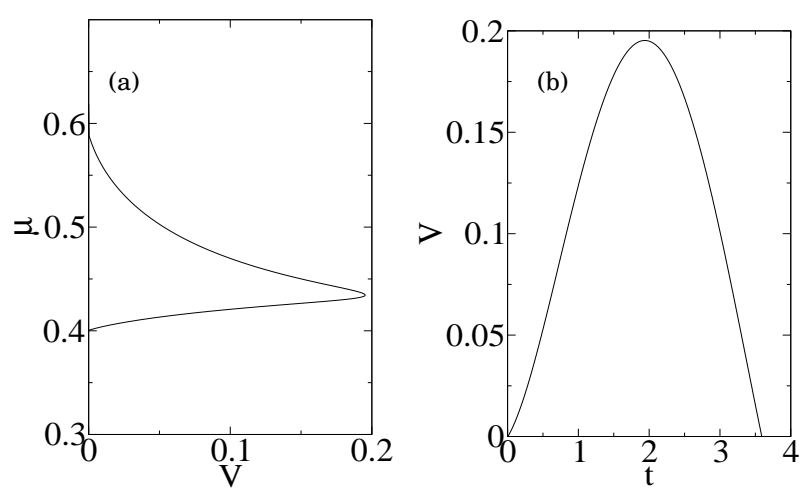

Figure 4. Unstable sliding of a spring-block system, described by equations (10) and (14), due to the static friction. (a) Velocity dependence of the friction coefficient during the unstable slip. (b) Temporal behavior of the block velocity. linear differential equation. Because the critical slip distance depends on the sliding velocity, it is more convenient to describe the evolution law with respect to time instead of the slip distance. The relaxation process is then described by the following evolution law.

$$
\dot{\mu}(t)=-\tau\left(\mu(t)-\mu_{\mathrm{ss}}\right),
$$

where $\mu_{\mathrm{ss}}$ is the stationary friction coefficient, which generally depends on the inertial number and other nondimensional parameters. We do not discuss a stationary constitutive law here (See, for example, [GDR MiDi, 2004; da Cruz et al. , 2005; Jop et al., 2004; Hatano, 2007]). It is essential to notice that equation (8) is length-free; i.e., the equation does not have any length constants. In the following section, we discuss some important consequences of this length-free evolution law.

\section{Discussions}

So far we have discussed the nature of the relaxation time in a transient process of granular layers and obtained the evolution law. In the rest of this paper, we discuss their important consequences in application to a more general situation. First, using equation (8), we describe the unstable sliding of a block on granular layers. The equation of motion is given as

$$
M \ddot{X}=-M \Omega^{2}\left(X-X_{0}\right)-\mu N,
$$

where $M$ is the mass of a block, $N$ is the normal load, $M \Omega^{2}$ is the stiffness of the spring, and $X_{0}$ is the equilibrium point of the spring. As the temporal evolution of the friction coefficient $\mu$ is given by equation (8), the block motion is determined combining these equations. Before explicitly solving Eqs. (8) and (9), we can see the essential property of the dynamics by dimensional analysis. Note that equation (9) has the time constant $\Omega^{-1}$ and the length constant $N / M \Omega^{2}$, whereas equation (8) does not have any length constants but the time constant $\tau$. This means that any lengths defined in the resulting dynamics, such as the critical slip distance, is scaled by $N / M \Omega^{2}$. Namely, the critical slip distance is not prescribed by the characteristic length of the microscopic geometry but more macroscopic parameters: the normal load and the stiffness of the spring. In order to see this more explicitly, we solve Eqs. (8) and (9). To this end, it is convenient to de-dimensionalize equation (9) as

$$
\Omega^{-2} \ddot{x}=-x-\mu,
$$

where $x \equiv\left(X-X_{0}\right) M \Omega^{2} / N$. The initial condition of the block motion is given as $x(0)=-\mu(0)$ and $\dot{x}(0)=0$. We assume that the slip instability is caused by the static friction; i.e., $\mu(0)=\mu_{s}$. This is easily realizable in granular layers if the initial state is sufficiently consolidated. Here we consider only $\dot{x}(t)>0$, i.e., we solve the block motion until it stops again.

The dynamics of the block is explicitly solvable if we assume that the dynamic friction coefficient is independent of the sliding velocity; i.e., $\mu_{\mathrm{SS}}=\mu_{d}$. Then the solution is given as

$$
\begin{aligned}
& x(t)=-\frac{\mu_{s}-\mu_{d}}{1+C^{2}}\left[C \sin \Omega t+\cos \Omega t+C^{2} e^{-t / \tau}\right]-\mu_{d} \\
& \mu(t)=\left(\mu_{s}-\mu_{d}\right) e^{-t / \tau}+\mu_{d}
\end{aligned}
$$

where $C \equiv \Omega \tau$ is the ratio of the two time constants in Eqs. (8) and (10). Then the the critical slip distance is apparent 
from Eqs. (11) and (12).

$$
D_{c} \simeq\left[x(\tau)+\mu_{s}\right] \frac{N}{M \Omega^{2}} .
$$

The numerical factor $\left[x(\tau)+\mu_{s}\right]$ is of the order of 1 unless $C \ll 1$. Note that $D_{c}$ is scaled with $N / M \Omega^{2}$, which is approximately equal to the slip distance. We wish to stress that, in granular layers, the critical slip distance is not scaled with the characteristic length of the microscopic geometry such as the surface roughness. This is the natural consequence of the legth-free nature of the relaxation, represented in the form of equation (3).

We also test a more plausible law for the dynamic friction, which is recently found in the DEM simulation.

$$
\mu_{\mathrm{SS}}=\mu_{0}+s I^{\phi}
$$

where $\phi \simeq 0.3$ and $s$ is a numerical factor of the order of 0.1 [Hatano, 2007]. Here we interpret $I$ as $V \sqrt{m / N d}$. The resulting dynamics is shown in Figure 4, where we adopt $\mu_{0}=0.3, s=0.2$, and $C=1$. The dynamics is very similar to that obtained in an experiment [Nasuno et al., 1998], in which the nondimensional number $C$ is estimated to be of the order of 1 .

Despite the feasibility in reproducing an experimental result on granular matter, we have to remark that the nondimensional parameter $C=\Omega \tau$ may be very small under a seismogenic condition. For example, if we assume that $P=100 \mathrm{MPa}, d=10 \mu \mathrm{m}$, and $H=1 \mathrm{~cm}$, using equation (6), the relaxation time $\tau$ is of the order of $10^{-4} \mathrm{~s}$. As the seismic slip takes place in seconds, $C$ is of the order of $10^{-4}$ so that the critical slip distance is negligible compared with the total slip distance. However, note that the framework of the length-free evolution law is not limited to the relaxation process of the velocity profile. It is straightforward to extend the present evolution law to incorporate any processes that affect the frictional strength. One of the most illustrating examples is a mechanochemical effect such as thermal decomposition of calcite, the rate constant of which is on the order of $1 \mathrm{sec}$ or even much larger depending on the temperature [Hirono et al. , 2007]. A plausible modeling is in progress and the result will be presented elsewhere.

Acknowledgments. The author gratefully acknowledges the discussion with Osamu Kuwano, Ryosuke Ando, Jean-Pierre Villote, and Pascal Bernard.

\section{References}

Aharonov, E. and D. Sparks (2002), Shear profiles and localization in simulations of granular materials, Phys. Rev. E 65, doi:051302, 10.1103/PhysRevE.65.051302.

Aki, K. (1987), Magnitude-frequency relation for small earthquakes: A clue to the origin of fmax of large earthquakes, $J$. Geophys. Res. 92, 1349-1355, doi:10.1029/JB092iB02p01349.

Cundall, P. A. and O. D. L. Strack (1979), A distinct element model for granular assemblies. Geotechnique, Geotechnique 29, 47-65. da Cruz, F., S. Emam, M. Prochnow, J. N. Roux, and F. Chevoir (2005), Rheophysics of dense granular materials: Discrete simulation of plane shear flows, Phys. Rev. E 72, 021309, doi:10.1103/PhysRevE.72.021309.

Dieterich, J. H. (1979), Modeling of rock friction 1. Experimental results and constitutive equations, J. Geophys. Res. 84(B5), 2161-2168, doi:10.1029/JB084iB05p02161.

Engelder, J. T. (1974), Cataclasis and the Generation of Fault Gouge, Bull. Geological Soc. Am. 85, 1515-1522, doi:10.1130/0016-7606(1974)85.

Garzó, V. and J. W. Dufty (1999), Dense fluid transport for inelastic hard spheres, Phys. Rev. E 59, 5895-5911, doi:10.1103/PhysRevE.59.5895.

GDR MiDi (2004), On dense granular flows, Euro. Phys. J. E 14, 367-371, doi:10.1140/epje/i2003-10153-0.

Hatano, T. (2007), Power-law friction in closely packed granular materials, Phys. Rev. E. 75, 060301(R), doi:10.1103/PhysRevE.75.060301.

Hazzard, J.F. and K. Mair (2003), The importance of the third dimension in granular shear, Geophys. Res. Lett. 30, 1708, doi:10.1029/2003GL017534.

Hirono, T., T. Yokoyama, Y. Hamada, W. Tanikawa, T. Mishima, M. Ikehara, V. Famin, M. Tanimizu, W. Lin, W. Soh, and S. Song (2007), A chemical kinetic approach to estimate dynamic shear stress during the 1999 Taiwan Chi-Chi earthquake, Geophys. Res. Lett. 34, L19308, doi: 10.1029/2007GL030743.

Ida, Y. (1973), The maximum acceleration of strong ground motion, Bull. Seism. Soc. Am. 63, 959-968.

Ide, S., and M. Takeo (1997), Determination of constitutive relations of fault slip based on seismic wave analysis, J. Geophys. Res. 102(B12), 27379-27391, 10.1029/97JB02675.

Jop, P., Y. Forterre, and O. Pouliquen (2006), A constitutive law for dense granular flows, Nature, 441, 727-730, doi:10.1038/nature04801.

Marone, C. and B. Kilgore (1993), Scaling of the critical slip distance for seismic faulting with shear strain in fault zones, Nature 362, 618-621, doi:10.1038/362618a0.

Marone, C. (1998), Laboratory-derived friction laws and their application to seismic faulting, Ann. Rev. Earth Planet. Sci. 26, 643-696, doi: 10.1146/annurev.earth.26.1.643.

Nasuno, S., A. Kudrolli, A. Bak, and J. P. Gollub (1998), Timeresolved studies of stick-slip friction in sheared granular layers, Phys. Rev. E. 58, 2161-2171, doi:10.1103/PhysRevE.58.2161.

Ohnaka, M., 2000, A physical scaling relation between the size of an earthquake and its nucleation zone size, Pure and Applied Geophysics 157, 2259-2282, doi: 10.1007/PL00001084.

Ohnaka, M., and L.-f. Shen (1999), Scaling of the shear rupture process from nucleation to dynamic propagation: Implications of geometric irregularity of the rupturing surfaces, J. Geophys. Res. 104, 817-844, doi:10.1029/1998JB900007.

Ohnaka, M. (2003), A constitutive scaling law and a unified comprehension for frictional slip failure, shear fracture of intact rock, and earthquake rupture, J. Geophys. Res. 108(B2), 2080, doi:10.1029/2000JB000123.

Savage, S. B. and K. Hutter (1989), The motion of a finite mass of granular material down a rough incline, J. Fluid. Mech. 199, 177-215, doi:10.1017/S0022112089000340.

Scholz, C. H. (1987), Wear and gouge formation in brittle faulting, Geology 15, 493-495, doi:10.1130/0091-7613(1987)15.

Scholz, C. H. (2002), The mechanics of earthquakes and faulting, (Cambridge University Press, Cambridge).

Takahiro Hatano, Earthquake Research Institute, University of Tokyo, 1-1-1 Yayoi, Bunkyo, Tokyo 113-0032, Japan (hatano@eri.u-tokyo.ac.jp) 